\title{
The Real Structure of $\mathrm{Cu}_{3} \mathrm{Ni}_{2} \mathrm{SbO}_{6}$ and $\mathrm{Cu}_{3} \mathrm{Co}_{2} \mathrm{SbO}_{6}$ Delafossites with Honeycomb Lattices by Aberration-Corrected HRTEM
}

\author{
R. Ramlau ${ }^{1}$, R. Schneider ${ }^{2}$, J. H. Roudebush ${ }^{3}$, and R. J. Cava ${ }^{3}$ \\ 1. Max Planck Institute for Chemical Physics of Solids, Dresden, Germany. \\ 2. Karlsruhe Institute of Technology, Laboratory for Electron Microscopy, Karlsruhe, Germany. \\ 3. Princeton University, Department of Chemistry, Princeton, NY 08544, USA.
}

The Delafossite $\left(\mathrm{CuFeO}_{2}\right)$ structure is formed from triangular layers of edge-sharing metal-oxygen octahedra separated by $\mathrm{Cu}^{1+}$ sticks. It resembles the $\mathrm{NaFeO}_{2}$ structure, with layers of $\mathrm{Cu}^{1+}$ sticks replacing the layers of $\mathrm{Na}^{+}$octahedra. Materials with such structures have been of interest as transparent films with p-type conductivity, thermoelectrics, superconductors, and multiferroics. Two polytypes are commonly found for Delafossites: The $2 H$ polytype, in which the axes of the octahedra are oriented $180^{\circ}$ in neighboring layers that are stacked in a hexagonal close packed fashion, and the $3 R$ polytype, in which all octahedra axes are oriented in the same direction, and the stacking is cubic close packed.

The crystal structures of two Delafossites, $\mathrm{Cu}_{3} \mathrm{Ni}_{2} \mathrm{SbO}_{6}$ and $\mathrm{Cu}_{3} \mathrm{Co}_{2} \mathrm{SbO}_{6}$, were determined by highresolution synchrotron powder X-ray diffraction [1]. The $\mathrm{Ni}$ and $\mathrm{Co}$ are ordered with respect to $\mathrm{Sb}$ in the layer of edge sharing octahedra, forming magnetic layers with honeycomb geometry. AC-HRTEM (together with SAED) confirms ordering and identifies examples of the stacking polytypes. Low temperature synthetic treatments result in disordered stacking of the layers, but heating just below their melting points results in nearly fully ordered stacking variants. The major variant in both cases is a monoclinic distortion of a hexagonal 6-layer Delafossite polytype (described as $2 \mathrm{M}$ or $6 H$ ), but a significant amount of a hexagonal 2-layer polytype $(2 \mathrm{H})$ is also present for the Ni case.

The Delafossite polytypes of $\mathrm{Cu}_{3} \mathrm{Ni}_{2} \mathrm{SbO}_{6}$ and $\mathrm{Cu}_{3} \mathrm{Co}_{2} \mathrm{SbO}_{6}$ under consideration do not exhibit differences in the stacking (with respect to the atom sites) of their $\mathrm{Cu}$ and mixed Ni/Sb or $\mathrm{Co} / \mathrm{Sb}$ honeycomb layers. The layer stacking is ...ABAC... - with the mixed layer always in position "A"and the axes of the metal octahedra are rotated by $180^{\circ}$ from one "A"-positioned layer to the next. The different polytypes are constituted by the stacking of the ordering scheme in the "A"-positioned layers, this means by the stacking $(\ldots \alpha \beta \gamma \ldots$ or ... $\alpha \beta \ldots)$ of Sb layers orderly decorated with $\mathrm{Ni}$ or Co.

HRTEM of $\mathrm{Cu}_{3} \mathrm{Ni}_{2} \mathrm{SbO}_{6}$ confirmed polytype $2 M$ and revealed the existence of polytype $2 H$. The latter occurs in extended domains, which are intergrown with the primary monoclinic polytype. Moreover, the real structure phenomena comprise formation of twin domains within the $2 M$ polytype and occurrence of domains with exotic long-range stacking order built up of 10 layers in the repetition unit.

Conventional TEM showed stacking faults, either as isolated or consecutive planes, in high density. Again, these stacking faults do not involve layer reorientations, but rather, within the general layer sequence, the shift of the layers to move the one metal (i.e. Ni) to a position where the other (i.e. Sb) would normally be expected - only the decoration of the layers is altered in the stacking faults. In rare cases aa-stacking of two neighboring Ni/Sb layers was found. It seems possible that within a honeycomb layer, 2-dimensional ordering domains are formed that are in anti-phase position to each other. Then the stacking scheme would vary from place to place and it can be understood why some stacking faults do not extend over the whole plane. 
Reference:

[1] J. H. Roudebush et al., Inorganic Chemistry 52 (2013) 6083-6095.
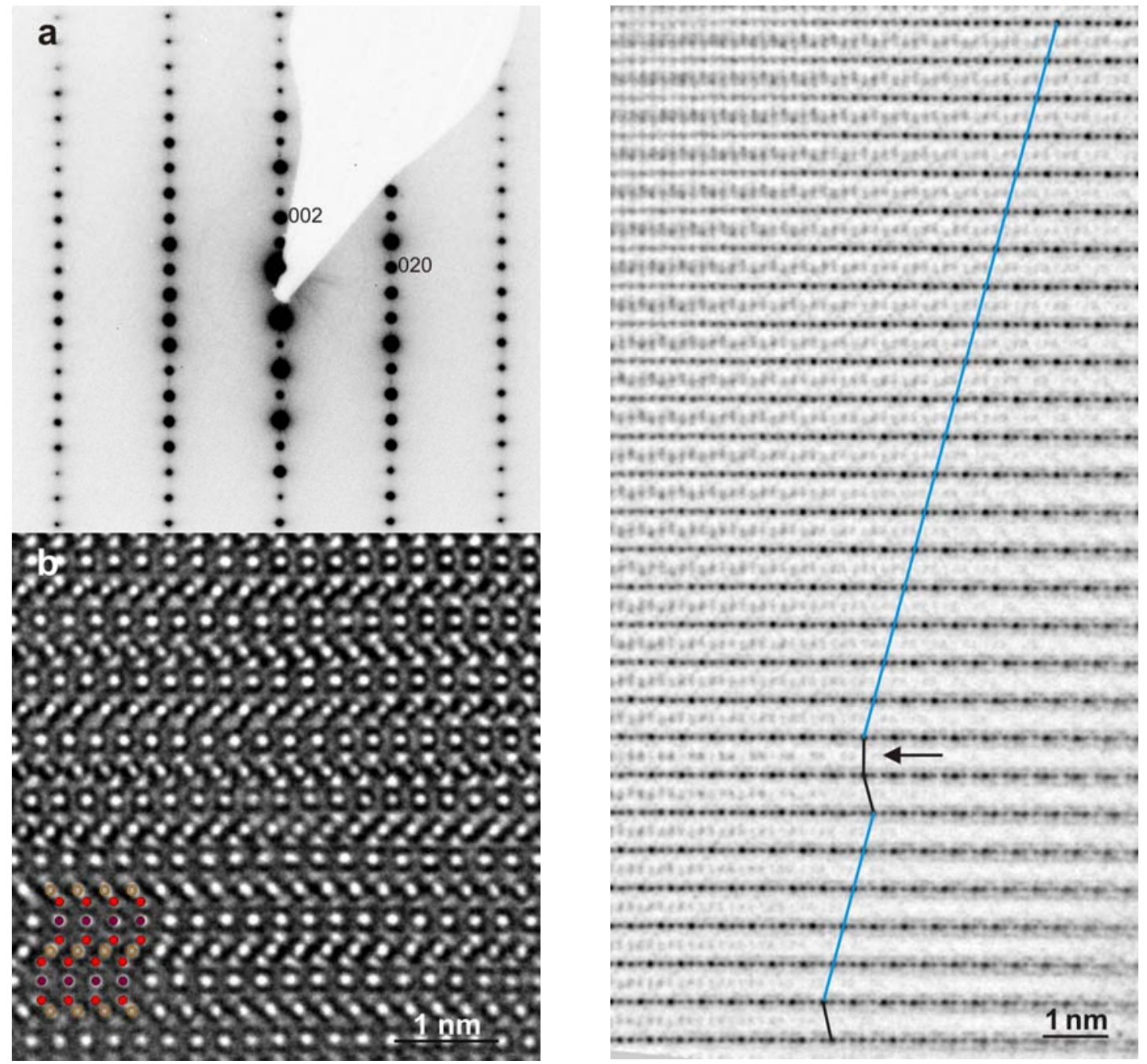

Figure 1 (left). (a) SAED pattern of the $\mathrm{Cu}_{3} \mathrm{Ni}_{2} \mathrm{SbO}_{6} 2 M$-polytype in [100] $]_{m}$ crystal orientation (space group: $C 2 / c$; crystallographic data given in [1]) and (b) AC-HRTEM image in which columns of $\mathrm{Ni} / \mathrm{Sb}$, $\mathrm{Cu}$ and $\mathrm{O}$ atoms are clearly resolved (FEI Titan ${ }^{3} 80-300$ with $C_{S}$ image corrector). The overlay in (b) shows the corresponding projection of the crystal structure; $\mathrm{Cu}$ : purple, Oxygen: red, $\mathrm{Ni}$ : orange, $\mathrm{Sb}$ : silver. Honeycomb layers ( $\mathrm{Cu}$ and $\mathrm{Ni} / \mathrm{Sb}$; purple and orange/silver) are stacked in ....ABAC... sequence.

Figure 2 (right). AC-HRTEM image in the $[110]_{m}$ zone axis of $\mathrm{Cu}_{3} \mathrm{Ni}_{2} \mathrm{SbO}_{6}-2 M$ where the monoclinic ... $\alpha \beta \gamma \ldots$ stacking of the "orderly decorated" $\mathrm{Ni} / \mathrm{Sb}$ layer is highlighted with a blue line. The mixed layer is emphasized by an appropriate choice of focus, the larger dot being Sb. Stacking faults occur in the lower third of the image, one of them shows an $\alpha \alpha$-stacking of two neighboring $\mathrm{Ni} / \mathrm{Sb}$ layers (arrow). Image contrast was inverted for reproduction! 